\begin{tabular}{|c|c|}
\hline Title & Charge Density Gradients of Polymer Thin Film by Gaseous Phase Quaternization \\
\hline Author(s) & Ko, Y eongun; Christau, Stephanie; von Klitzing, Regine; Genzer, Jan \\
\hline Citation & $\begin{array}{l}\text { A CS macro letters, 9(2), 158-162 } \\
\text { https://doi.org/10.1021/acsmacrolett.9b00930 }\end{array}$ \\
\hline Issue Date & $2020-02-18$ \\
\hline Doc URL & http:/hdl.handle.net/2115/80476 \\
\hline Rights & $\begin{array}{l}\text { This document is the A ccepted Manuscript version of a Published Work that appeared in final form in ACS macro } \\
\text { letters, copyright c A merican Chemical Society after peer review and technical editing by the publisher. To access the } \\
\text { final edited and published work see https://pubs.acs.org/doi/10.1021/acsmacrolett.9b00930. }\end{array}$ \\
\hline Type & article (author version) \\
\hline File Information & Charge Density Gradients of Polymer Thin Film by Gaseous Phase Quaternization.pdf \\
\hline
\end{tabular}

Instructions for use 


\title{
Charge density gradients of polymer thin film by gaseous phase quaternization
}

\author{
Yeongun $\mathrm{Ko}^{1}$, Stephanie Christau ${ }^{2}$, Regine von Klitzing ${ }^{3}$, Jan Genzer ${ }^{1,4, *}$ \\ ${ }^{1}$ Department of Chemical and Biomolecular Engineering, North Carolina State University \\ Raleigh, NC 27695-7905, USA \\ 2 Department of Chemical Engineering, Biointerfaces Institute, University of Michigan \\ Ann Arbor, MI 48109, USA \\ ${ }^{3}$ Department of Physics, Technische Universität Darmstadt \\ 64289 Darmstadt, Germany \\ ${ }^{4}$ Global Station for Soft Matter, Global Institution for Collaborative Research and Education \\ Hokkaido University, Sapporo, 060-0808, Japan
}

\begin{abstract}
We report on the rapid formation of charge density gradients in polymer films by exposing poly([2dimethylaminoethyl] methacrylate) (PDMAEMA) films resting on flat silica substrates to methyl iodide (i.e., MI, also known as iodomethane) vapors. We adjust the charge gradient by varying the MI concentration in solution and the process time. The thickness of the parent PDMAEMA film does not affect the diffusion of MI through and the reaction kinetics in the films. Instead, the diffusion of MI through the gaseous phase constitutes the limiting step in the overall process.
\end{abstract}

Charged polymers represent an important class of materials, whose chemical and physical properties get tailored by varying, e.g., the distance between charged repeat units or chain topology, leading to variations in diffusion or relaxation dynamics, glass transition, counter ion exchange, and other properties. ${ }^{1-3}$ Tertiary amines are often employed as parent polymers for postpolymerization modification reactions because they can be tailored to feature zwitterionic, strongly-charged units (after appropriate betanization or quaternization post-polymerization reactions, respectively) or can be weakly-charged in solutions with low $\mathrm{pH}^{4-9}$

Many researchers have studied the performance of polyelectrolyte thin films due to a

*Corresponding author: jgenzer@ncsu.edu 
variety of potential applications, i.e., lubrication, antifouling or antibacterial properties, or cell adhesion, to name a few. ${ }^{10-19}$ Gradients of chemical or physical properties in films enable investigating a given phenomenon of interest in a systematic fashion, thus reducing systematic experimental errors as well as laborious efforts in preparing individual samples while allowing a comprehensive and systematic survey of sample characteristics. ${ }^{11,20-23}$ Substrate-bound material gradients may also act as "engines" that provide a driving force for cell migration or droplet transport. $^{24-29}$ Several research groups prepared charge density gradients and studied their properties. Higgins and coworkers have focused on fabricating charge density gradients using small-molecule organosilanes. ${ }^{30-32}$ Although the gradients were well characterized, the organosilanes possess a few disadvantages, i.e., substrate-dependence, limited chemistry, limited thicknesses, and complex assemblies at interfaces. ${ }^{33-35}$ Lee and coworkers, demonstrated charge density gradients of polymer grafts using corona discharge. ${ }^{36,37}$ Braun and coworkers developed a method to form micrometer-scale gradients of charged polymer brushes by utilizing microfluidics. ${ }^{38,39}$ Very recently, Sun et al. ${ }^{40}$ reported on fabricating surface charge density gradients on surfaces by using fluorinated silica nanospheres and using water droplets delivered from different heights above the substrate to form substrate-dependent charge gradients.

(a)

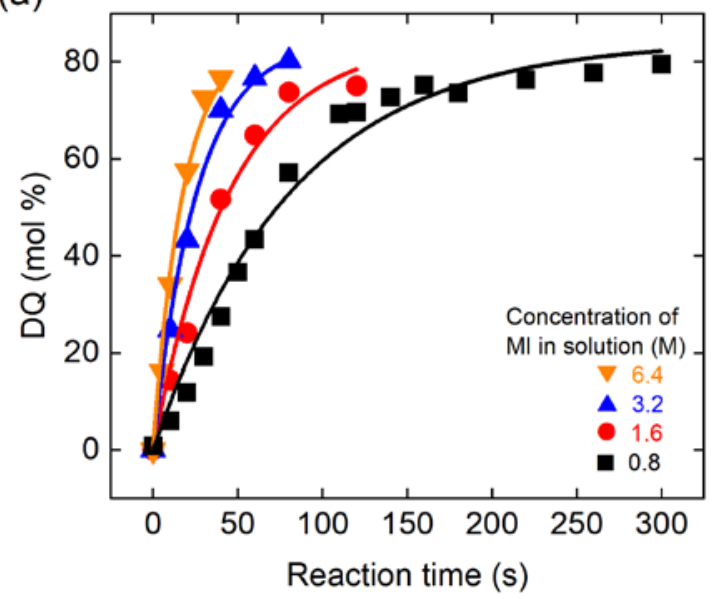

(b)

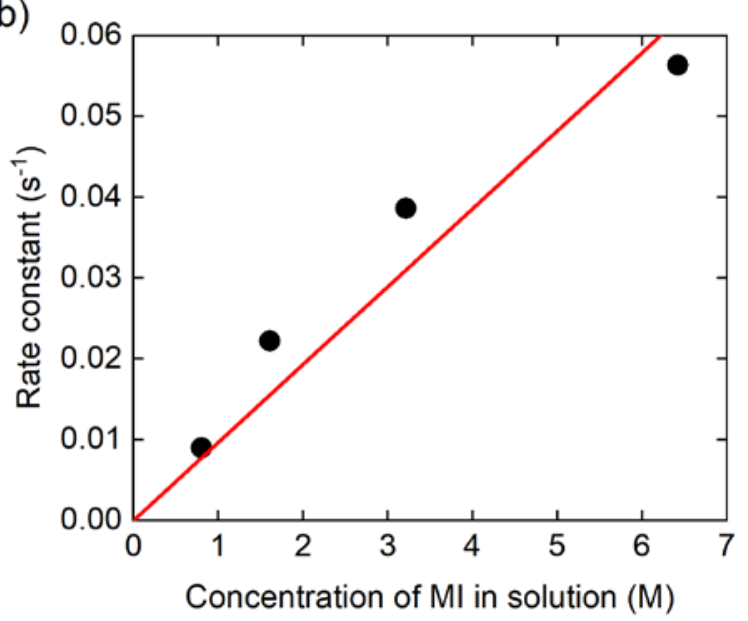

Figure 1. (a) Degree of quaternization (DQ) vs. reaction time in ethanol solution with variable methyl iodide (MI) concentration at ambient condition; (b) the rate constant for pseudo-first-order reaction with different MI concentration. The slope, $0.0097(\mathrm{M} \cdot \mathrm{s})^{-1}$, corresponds to the rate constant of the second-order reaction $\left(k_{2 l}, c f\right.$. Equation 1). The dry thickness of the PDMAEMA brushes was $\sim 93 \mathrm{~nm}$ as measured by ellipsometry at $100^{\circ} \mathrm{C}$. 
Here, we describe a simple method to form charge density gradients in poly([2dimethylaminoethyl] methacrylate) (PDMAEMA) thin films on flat silica substrates by vapor diffusion of methyl iodide (MI). The process is rapid: at $6.4 \mathrm{M}$ of MI concentration in solution, it takes only 40 seconds to quaternize PDMAEMA films to reach DQ 77 mol\%, which is comparable to the maximum DQ obtained in solution ( $\sim 55$ mol\%). We form the PDMAEMA layers by either surface-initiated polymerization or deposit PDMAEMA homopolymer films on the substrate by dip-coating (see supporting information, SI, for details). We lower the PDMAEMA-coated wafer vertically into a standard $50 \mathrm{~mL}$ beaker (inner diameter $4 \mathrm{~cm}$, height $5.5 \mathrm{~cm}$ ) and inject MI solution so that the sample contacts barely the solution level. As MI evaporates from the solution, it deposits onto and reacts with the tertiary amines in the DMAEMA units in the films. The latter process forms quaternized (i.e., positively charged) groups in the PDMAEMA films (Scheme S1). For each concentration of MI in solution, we adjust the spatial distribution of the charged groups in the substrates by varying the process time (i.e., diffusion of MI in the vapor and through the film and the reaction).

We first studied the quaternization kinetics in the liquid phase by immersing PDMAEMA brush samples into solutions featuring different concentrations in ethanol (i.e., 0.8, 1.6, 3.2, and $6.4 \mathrm{M}$ ) for various times. We assumed that the quaternization in the liquid phase is reaction limited given that the diffusion of MI from bulk liquid solution (D $\sim 10^{-5} \mathrm{~cm}^{2} / \mathrm{sec}$ ) was very fast even in thick films $(\sim 300 \mathrm{~nm}){ }^{6,8}$ We measured the dry thickness of quaternized PDMAEMA (qPDMAEMA) brushes and the degree of quaternization (DQ) using ellipsometry by implementing the method we recently described. ${ }^{41}$ The index of refraction of the films increases linearly with increasing DQ. ${ }^{41}$ (see SI).

Previously, researchers used a second-order reaction kinetic model to characterize quaternization reactions. ${ }^{4,42}$ Because in our system, MI is present in large excess, we fit the experimental data using pseudo-first-order reaction kinetics (Equations 1, 2, and Figure 1a).

$$
\begin{aligned}
& \frac{d n}{d t}=-k_{2 l} \cdot n \cdot c \cong-k_{1 l} \cdot n \\
& \operatorname{DQ}(\operatorname{mol} \%) \equiv 100 \% \times \frac{n_{0}-n}{n_{0}}=A \times\left[1-\exp \left(-k_{1 l} \cdot t\right)\right]
\end{aligned}
$$

$n_{0}, n, c, k_{1 l}, k_{2 l}, t$ and $A$ represent the initial concentration of unmodified monomer, the concentrations of unmodified monomer, and MI, respectively, the rate constants for the pseudo- 
first-order reaction ( $\left.k_{1 l} \cong k_{2 l} \cdot c\right)$, the second-order reaction in the liquid phase, respectively, the process time, and the upper limit of DQ. The upper limit of DQ was found to be $\sim 85 \mathrm{~mol} \%$ which is in good agreement with the result reported Přádný et al. who studied bulk quaternization kinetics of PDMAEMA. ${ }^{4}$ Two major effects may influence the upper limit of quaternization: 1) steric hindrance due to the presence of two bulky methyl groups attached to nitrogen in DMAEMA units, ${ }^{43}$ 2) decreasing solubility of qPDMAEMA in ethanol with increasing DQ, ${ }^{8}$ accompanied with the associated chain collapse, which limits the reactivity of the remaining unmodified DMAEMA units in the random copolymer with the quaternizing agent.

We fit the experimental data to Equation 2 and plot the $k_{1 l}$ values against different MI solution concentrations in Figure 1b, which displays a linear dependence of $k_{1 l}$ on the MI concentration in solution (see SI). We then estimate the rate constant for the second-order reaction kinetics, $k_{2 l} \sim 0.0097(\mathrm{M} \cdot \mathrm{s})^{-1}$, by using the slope in the linear fit in Figure 1b. The obtained value is a little larger than the literature value $\sim 0.003(\mathrm{M} \cdot \mathrm{s})^{-1}$, measured in bulk reaction. ${ }^{4,42}$ We explain the difference by assuming that our system involves surface reaction, which features highly concentrated reactive centers compared to the bulk polymer solution. ${ }^{44}$ Compared to free polymers in bulk solutions, the swollen brushes exhibit significantly higher local concentration of the reactive repeat units, which accelerates the reaction kinetics.

(a)

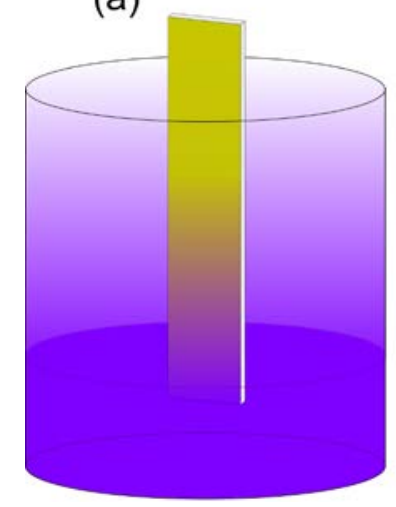

(b)

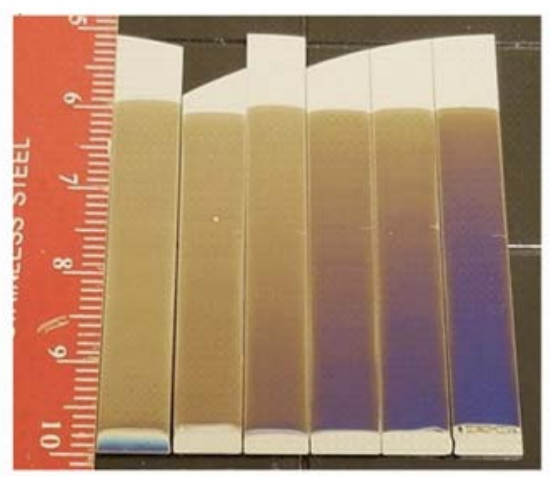

Figure 2. (a) Experimental setup used to produce charge density gradients in polymer films by vapor diffusion of methyl iodide. (b) Quaternized PDMAEMA films formed after exposure to methyl iodide vapors (the units in the ruler are centimeters). The process time was 0 (i.e., just before MI solution injection), 15, 30, 45, 60, and 90 sec from left to right. The concentration of methyl iodide in ethanol solutions was $3.2 \mathrm{M}$. The dry thickness of the parent PDMAEMA brushes was $\sim 70 \mathrm{~nm}$ as measured by ellipsometry at $100^{\circ} \mathrm{C}$. 
We prepared samples featuring charge density gradients of polymer thin films by reacting parent PDMAEMA films with MI vapors ( $c f$. Figure 2). Silicon wafers $(6 \mathrm{~mm} \times 40 \mathrm{~mm})$ coated with PDMAEMA films were placed vertically into an empty standard $50 \mathrm{~mL}$ glass beaker (inner diameter $4 \mathrm{~cm}$, height $5.5 \mathrm{~cm})$. MI solutions in ethanol (200 $\mu \mathrm{L})$ were poured into the beaker so that the bottom portion of the sample ( 2-3 mm) was immersed partially in the MI solution. After a given time, the sample was removed from the beaker, dried by flowing nitrogen gas, and the sample refractive index was assessed by ellipsometry. The entire process includes evaporation of MI from solution, diffusion through the air, diffusion through the polymer layer, and reaction with tertiary amines in PDMAEMA. We form charge density gradients in the parent films by using solutions of various MI concentrations and process times.

(a)

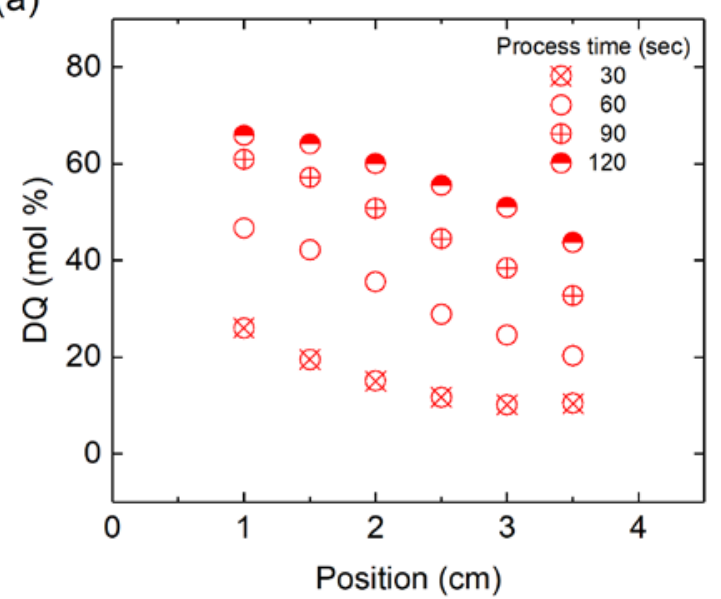

(b)

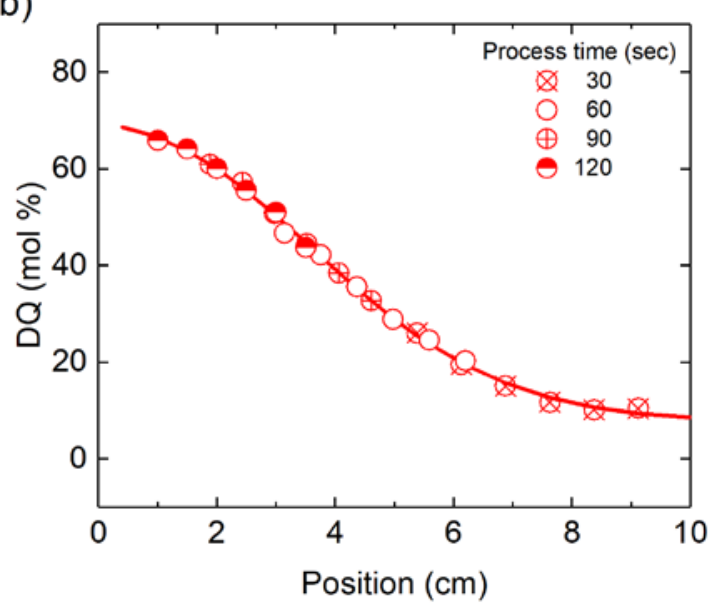

Figure 3. (a) DQ of charge density gradient films with different process time before data shifting. The quaternization in the gaseous phase was achieved with 1.6 M MI in ethanol. (b) The corresponding data after a horizontal shift. The solid curve is the result of fitting the experimental data to Equation 5 with a constant $D=0.13 \mathrm{~cm}^{2} / \mathrm{sec}$ and $t=120 \mathrm{sec}$. The shift values are reported in the SI.

We measure the DQ (from the variation of the refractive index at $600 \mathrm{~nm}$ ) as a function of the position on the sample for various process times. Figure 3 plots examples of such spatiotemporal profiles for solution concentration of $\mathrm{MI}$ in ethanol of $1.6 \mathrm{M}$. We pick the data set collected at $120 \mathrm{sec}$ as a basis and shift the DQ vs. position data in the sample prepared at $90 \mathrm{sec}$ by the same amount along the horizontal axis so that the DQ from both specimens fall onto a master plot. We repeat the same procedure for the remaining data sets. Thus, each data set possesses a unique shift factor. Figure 4S in the SI reports shift factors for all samples studied. From the data in Figure 4a, which features time shifts from solutions having four different 
concentrations of MI in ethanol, we notice that samples prepared by quaternization from solutions having a lower concentration of MI exhibited broader DQ gradients in the films. It indicates that, similar to the reaction in the liquid phase, the MI concentration in vapor influences the kinetics of quaternization. We analyzed the acquired gradient data with the following equations:

$$
\begin{aligned}
& \frac{d n}{d t}=-k_{2 g} \cdot n \cdot c(z, t) \\
& c(z, t)=c(0, t) \cdot \operatorname{erfc}\left(\frac{z}{2 \sqrt{D \cdot t}}\right) \\
& \mathrm{DQ}(\operatorname{mol} \%)=A \times\left\{1-\exp \left\{-k_{1 g} \cdot\left[t \cdot\left(\operatorname{erfc}\left(\frac{z}{2 \sqrt{D t}}\right)-\frac{z \cdot e^{-z^{2} / 4 D t}}{\sqrt{\pi \cdot D t}}\right)+\right.\right.\right. \\
& \left.\left.\left.\frac{z^{2} \cdot \operatorname{erfc}\left(\frac{z}{2 \sqrt{D t}}\right)}{2 D}\right]\right\}\right\}
\end{aligned}
$$

where $z, k_{1 g}, k_{2 g}$, and $D$ are the position, the rate constants for the pseudo-first-order reaction and the second-order reaction in the gaseous phase, and diffusivity, respectively.

(a)

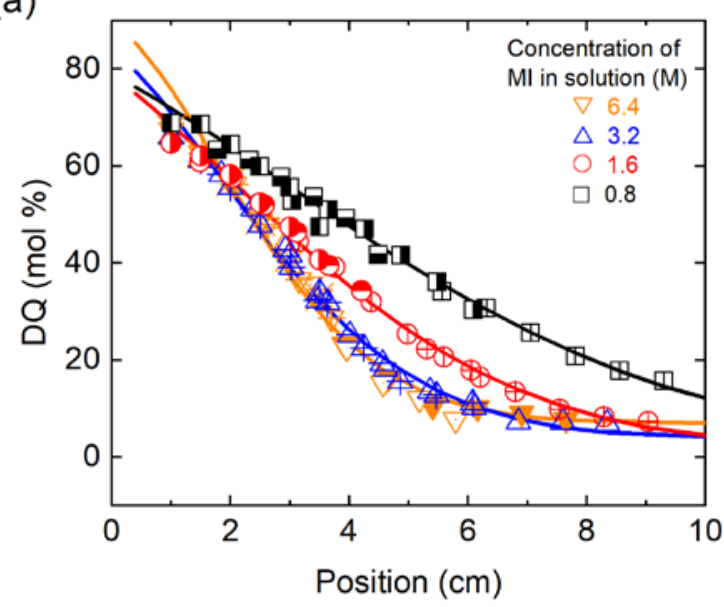

(b)

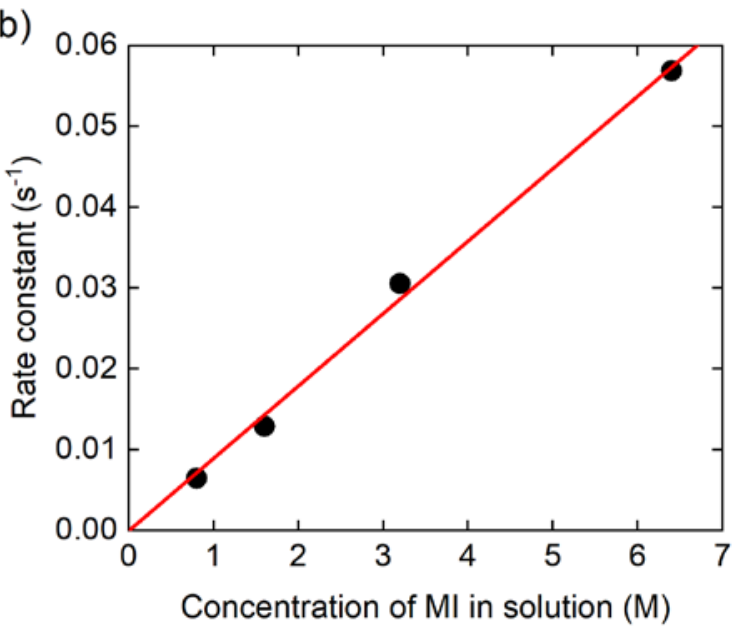

Figure 4. (a) Quaternization gradient with a different nominal concentration of MI in an ethanol solution. The process times for various concentrations are as follows; $\mathbb{X}: 10, \nabla: 20, \forall: 30, \nabla: 40$ sec for $6.4 \mathrm{M}$,

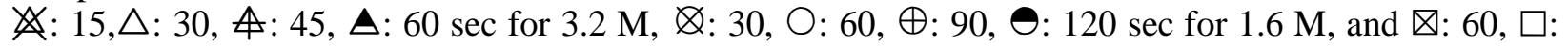
120, $\boxplus: 180$, $\square: 240 \mathrm{sec}$ for $0.8 \mathrm{M}$. Dry thicknesses of PDMAEMA films were $141 \mathrm{~nm}$. The DQ vs. position plots have been obtained by shifting horizontally the data collected at various times (see SI). The symbols represent measured data, and the lines are obtained by fitting the data to Equation 5. (b) Rate constants obtained from fitting with different nominal concentrations of MI in solution. The slope is 0.009 $(\mathrm{M} \cdot \mathrm{s})^{-1}$. 
Equation 4 allows estimating the MI concentration in the gaseous phase at a certain position and time. The diffusivity of $\mathrm{MI}$ in the air is $0.13 \mathrm{~cm}^{2} / \mathrm{sec}^{45}$ We assume that $\mathrm{MI}$ is the excess reagent, and the interfacial concentration of MI, $c(0, t)$, remains constant throughout the process. We solve Equation 3 and substitute Equation 4 to obtain Equation 5, in which $k_{1 g}=k_{2 g} * c(0, t)$. We fit all experimental data using Equation 5 and plot $k_{1 g}$ in Figure $\mathbf{4 b}$ as a function of the concentration of MI in solution. The $k_{1 g}$ values are nearly identical to $k_{1 l}$. The $k_{1 g}$ changes linearly with the concentration of MI in solution. While we cannot estimate $k_{2 g}$ due to the unknown value of $c(0, t)$, we hypothesize that $k_{2 g}$ is higher than $k_{2 l}$ because the concentration of MI in the gaseous phase should be lower than that in the liquid. In the SI document, we provide evidence that our assumption is correct.

(a)

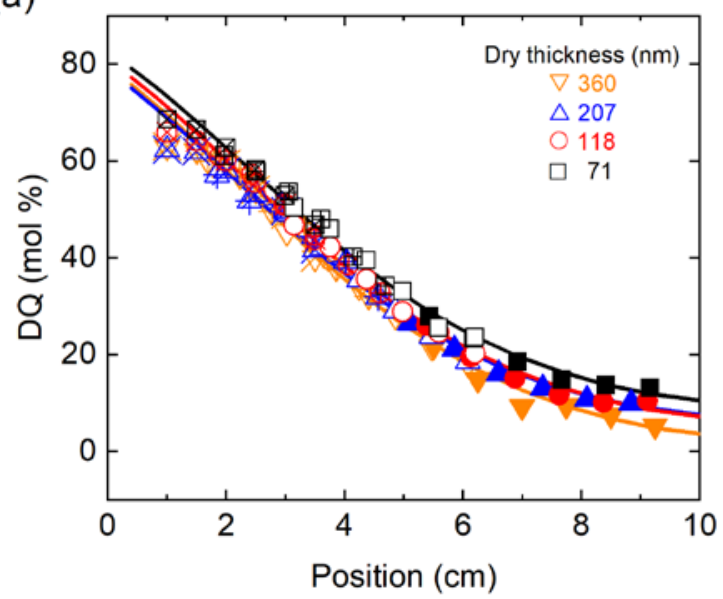

(b)

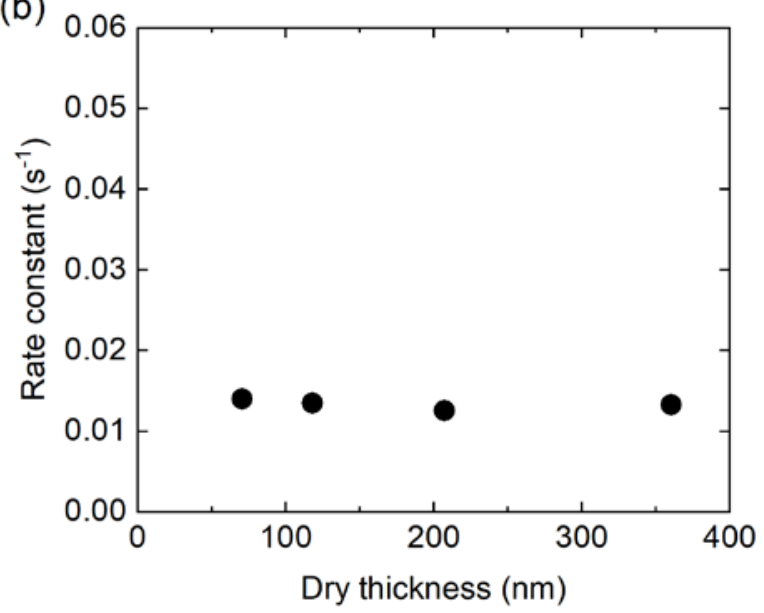

Figure 5. (a) Quaternization gradient with different PDMAEMA film thicknesses. The process times for

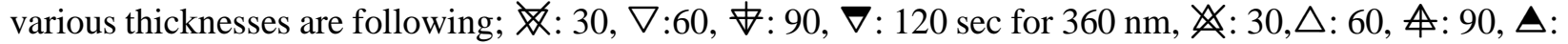
$120 \mathrm{sec}$ for $207 \mathrm{~nm}, \mathbb{:}: 30, \bigcirc: 60, \oplus: 90, \ominus: 120 \mathrm{sec}$ for $118 \mathrm{~nm}$, and $\otimes: 30, \square: 60, \boxplus: 90, \square: 120 \mathrm{sec}$ for $71 \mathrm{~nm}$. All modifications were carried out with 1.6 M of MI in an ethanol solution. The lines are obtained by fitting the data to Equation 5. (b) Rate constants obtained from fitting with different dry thicknesses of polymer films.

The diffusion of MI involves two distinguishable processes: 1) transport through the gaseous phase, and 2) diffusion through the polymer layer resting on the substrate. To gain insight into which diffusion process controls the entire kinetics, we repeated the experiments with PDMAEMA films of thicknesses ranging from 71 to $360 \mathrm{~nm}$ (Figure 5). The results plotted in Figure $5 \mathbf{b}$ show that all $k_{1 g}$ values are the same regardless of the initial thickness of the parent 
PDMAEMA film, which implies that the reaction kinetics is not affected by the PDMAEMA thicknesses.

In summary, we examined the quaternization kinetics of PDMAEMA films by MI vapors and modeled them with a pseudo-first-order reaction kinetic model. The reaction kinetics is very fast (i.e., it takes $\sim 40$ seconds to achieve DQ $\sim 77 \mathrm{~mol} \%$ ). The thickness of the polymer layer does not affect the diffusion in the studied thickness range or the reaction kinetics. The independency of the film thickness on the entire process implies that vapor transport is the limiting process, similar to vapor deposition of organosilanes onto flat silica substrates. ${ }^{46-48}$ The linearity between the shift factors and the square root of process time (cf. Figure S4) supports this claim. We thus suggest that the overall process is governed primarily by methyl iodide diffusion through the gaseous phase. We note that the "steepness" of the gradient depends on the height of the beaker. In the experiments described here, we used a standard $50 \mathrm{~mL}$ beaker. Using a shorter beaker one can produce sharper gradients; see SI for details.

A major advantage of this vapor deposition set up (besides its fast kinetics) is that it applies to both grafted and non-grafted polymer films. In the pseudo-first-order category of systems, position-dependent and gradually-varying degree of quaternization in free films offers a sound platform for ion transport studies, combinatorial investigation of optical and thermal (refractive index, glass transition) properties of thin films, and other phenomena. One may use this process in multi-step manufacture of complex combinatorial polymer coatings that feature a directional variation of other properties, i.e., position-dependent grafting density or molecular weight of polymer brushes.

\section{Associated content}

\section{Supporting information}

The Supporting Information is available free of charge on the ACS Publications website.

Preparation and characterization of polymer films. Temperature sweep of PMMA layers. Film thickness for different polymer solution concentration. Data shifting process (PDF).

\section{Author information}


*Email: jgenzer@ncsu.edu

ORCID: Yeongun Ko: 0000-0001-5770-6707

ORCID: Stephanie Christau: 0000-0003-1152-5606

ORCID: Regine von Klitzing: 0000-0003-0555-5104

ORCID: Jan Genzer: 0000-0002-1633-238X

\section{Acknowledgment}

The work was supported by the National Science Foundation, Grant no. DMR-1404639 and by the German Research Council (DFG) via the IRTG 1524. We appreciate the partial support from the National Science Foundation, Grant no. DMR-1809453. 


\section{References}

(1) Eisenberg, A.; Farb, H.; Cool, L. G. Glass Transitions in Ionic Polymers. J. Polym. Sci. Part A-2 Polym. Phys. 1966, 4, 855-868.

(2) Muthukumar, M. 50th Anniversary Perspective: A Perspective on Polyelectrolyte Solutions. Macromolecules 2017, 50, 9528-9560.

(3) Polymeropoulos, G.; Zapsas, G.; Ntetsikas, K.; Bilalis, P.; Gnanou, Y.; Hadjichristidis, N. 50th Anniversary Perspective: Polymers with Complex Architectures. Macromolecules 2017, 50, 1253-1290.

(4) Přádny, M.; Ševčík, S.; Vlček, P. Precursors of Hydrophilic Polymers. Polym. Bull. 1984, 12, 337-342.

(5) Bütün, V.; Armes, S. P.; Billingham, N. C. Selective Quaternization of 2(Dimethylamino)Ethyl Methacrylate Residues in Tertiary Amine Methacrylate Diblock Copolymers. Macromolecules 2001, 34, 1148-1159.

(6) Sanjuan, S.; Perrin, P.; Pantoustier, N.; Tran, Y. Synthesis and Swelling Behavior of PHResponsive Polybase Brushes. Langmuir 2007, 23, 5769-5778.

(7) Cheng, N.; Bao, P.; Evans, S. D.; Leggett, G. J.; Armes, S. P. Facile Formation of Highly Mobile Supported Lipid Bilayers on Surface-Quaternized PH-Responsive Polymer Brushes. Macromolecules 2015, 48, 3095-3103.

(8) Galvin, C. J.; Genzer, J. Swelling of Hydrophilic Polymer Brushes by Water and Alcohol Vapors. Macromolecules 2016, 49, 4316-4329.

(9) Ko, Y.; Genzer, J. Spontaneous Degrafting of Weak and Strong Polycationic Brushes in Aqueous Buffer Solutions. Macromolecules 2019, 52, 6192-6200.

(10) Richert, L.; Lavalle, P.; Vautier, D.; Senger, B.; Stoltz, J.-F.; Schaaf, P.; Voegel, J.-C.; Picart, C. Cell Interactions with Polyelectrolyte Multilayer Films. Biomacromolecules 2002, 3, 1170-1178.

(11) Kim, M. S.; Khang, G.; Lee, H. B. Gradient Polymer Surfaces for Biomedical Applications. Prog. Polym. Sci. 2008, 33, 138-164.

(12) Gribova, V.; Auzely-Velty, R.; Picart, C. Polyelectrolyte Multilayer Assemblies on Materials Surfaces: From Cell Adhesion to Tissue Engineering. Chem. Mater. 2012, 24, 854-869.

(13) Galvin, C. J.; Genzer, J. Applications of Surface-Grafted Macromolecules Derived from Post-Polymerization Modification Reactions. Prog. Polym. Sci. 2012, 37, 871-906.

(14) Krishnamoorthy, M.; Hakobyan, S.; Ramstedt, M.; Gautrot, J. E. Surface-Initiated Polymer Brushes in the Biomedical Field: Applications in Membrane Science, Biosensing, Cell Culture, Regenerative Medicine and Antibacterial Coatings. Chem. Rev. 2014, 114, 1097611026.

(15) Mi, L.; Jiang, S. Integrated Antimicrobial and Nonfouling Zwitterionic Polymers. Angew. Chemie Int. Ed. 2014, 53, 1746-1754.

(16) Zhang, W.; Zhao, Q.; Yuan, J. Porous Polyelectrolytes: The Interplay of Charge and Pores for New Functionalities. Angew. Chemie Int. Ed. 2018, 57, 6754-6773. 
(17) Li, A.; Ramakrishna, S. N.; Nalam, P. C.; Benetti, E. M.; Spencer, N. D. Stratified Polymer Grafts: Synthesis and Characterization of Layered "Brush" and "Gel” Structures. Adv. Mater. Interfaces 2014, 1, 1-8.

(18) Séon, L.; Lavalle, P.; Schaaf, P.; Boulmedais, F. Polyelectrolyte Multilayers: A Versatile Tool for Preparing Antimicrobial Coatings. Langmuir 2015, 31, 12856-12872.

(19) Tomlinson, M. R.; Genzer, J. Formation and Properties of Multivariant Assemblies of Surface-Tethered Diblock and Triblock Copolymers. Polymer 2008, 49, 4837-4845.

(20) Morgenthaler, S.; Zink, C.; Spencer, N. D. Surface-Chemical and -Morphological Gradients. Soft Matter 2008, 4, 419-434.

(21) Genzer, J.; Bhat, R. R. Surface-Bound Soft Matter Gradients. Langmuir 2008, 24, 2294 2317.

(22) Genzer, J. Surface-Bound Gradients for Studies of Soft Materials Behavior. Annu. Rev. Mater. Res. 2012, 42, 435-468.

(23) Collinson, M. M.; Higgins, D. A. Organosilane Chemical Gradients: Progress, Properties, and Promise. Langmuir 2017, 33, 13719-13732.

(24) Chaudhury, M. K.; Whitesides, G. M. How to Make Water Run Uphill. Science 1992, 256, 1539-1541.

(25) Ichimura, K.; Oh, S.-K.; Nakagawa, M. Light-Driven Motion of Liquids on a Photoresponsive Surface. Science 2000, 288, 1624-1626.

(26) Smith, J. T.; Tomfohr, J. K.; Wells, M. C.; Beebe, T. P.; Kepler, T. B.; Reichert, W. M. Measurement of Cell Migration on Surface-Bound Fibronectin Gradients. Langmuir 2004, 20, 8279-8286.

(27) Smith, J. T.; Elkin, J. T.; Reichert, W. M. Directed Cell Migration on Fibronectin Gradients: Effect of Gradient Slope. Exp. Cell Res. 2006, 312, 2424-2432.

(28) Ito, Y.; Heydari, M.; Hashimoto, A.; Konno, T.; Hirasawa, A.; Hori, S.; Kurita, K.; Nakajima, A. The Movement of a Water Droplet on a Gradient Surface Prepared by Photodegradation. Langmuir 2007, 23, 1845-1850.

(29) Jindan, W.; Zhengwei, M.; Huaping, T.; Lulu, H.; Tanchen, R.; Changyou, G. Gradient Biomaterials and Their Influences on Cell Migration. Interface Focus 2012, 2, 337-355.

(30) Ashraf, K. M.; Giri, D.; Wynne, K. J.; Higgins, D. A.; Collinson, M. M. Cooperative Effects in Aligned and Opposed Multicomponent Charge Gradients Containing Strongly Acidic, Weakly Acidic, and Basic Functional Groups. Langmuir 2016, 32, 3836-3847.

(31) Ashraf, K. M.; Khan, M. R. K.; Higgins, D. A.; Collinson, M. M. PH and Surface Charge Switchability on Bifunctional Charge Gradients. Langmuir 2018, 34, 663-672.

(32) Bautista-Gomez, J.; Forzano, A. V; Austin, J. M.; Collinson, M. M.; Higgins, D. A. VaporPhase Plotting of Organosilane Chemical Gradients. Langmuir 2018, 34, 9665-9672.

(33) Onclin, S.; Ravoo, B. J.; Reinhoudt, D. N. Engineering Silicon Oxide Surfaces Using SelfAssembled Monolayers. Angew. Chemie Int. Ed. 2005, 44, 6282-6304.

(34) Steinrück, H.-G.; Schiener, A.; Schindler, T.; Will, J.; Magerl, A.; Konovalov, O.; Li 
Destri, G.; Seeck, O. H.; Mezger, M.; Haddad, J.; Deutsch, M.; Checco, A.; Ocko, B. M. Nanoscale Structure of Si/SiO2/Organics Interfaces. ACS Nano 2014, 8, 12676-12681.

(35) Steinrück, H.-G.; Will, J.; Magerl, A.; Ocko, B. M. Structure of N-Alkyltrichlorosilane Monolayers on Si(100)/SiO2. Langmuir 2015, 31, 11774-11780.

(36) Lee, J. H.; Lee, J. W.; Khang, G.; Lee, H. B. Interaction of Cells on Chargeable Functional Group Gradient Surfaces. Biomaterials 1997, 18, 351-358.

(37) Lee, J. H.; Khang, G.; Lee, J. W.; Lee, H. B. Platelet Adhesion onto Chargeable Functional Group Gradient Surfaces. J. Biomed. Mater. Res. 1998, 40, 180-186.

(38) Koo, H.-J.; Waynant, K. V; Zhang, C.; Braun, P. V. Polymer Brushes Patterned with Micrometer-Scale Chemical Gradients Using Laminar Co-Flow. ACS Appl. Mater. Interfaces 2014, 6, 14320-14326.

(39) Koo, H.-J.; Waynant, K. V; Zhang, C.; Haasch, R. T.; Braun, P. V. General Method for Forming Micrometer-Scale Lateral Chemical Gradients in Polymer Brushes. Chem. Mater. 2014, 26, 2678-2683.

(40) Sun, Q.; Wang, D.; Li, Y.; Zhang, J.; Ye, S.; Cui, J.; Chen, L.; Wang, Z.; Butt, H. J.; Vollmer, D.; Deng, X. Surface Charge Printing for Programmed Droplet Transport. Nat. Mater. 2019, 18, 936-941.

(41) Ko, Y.; Miles, J. R.; Genzer, J. Determining Water Sorption and Desorption in Thin Hydrophilic Polymer Films by Thermal Treatment. ACS Appl. Polym. Mater. 2019, 1, 2495-2502.

(42) Přádný, M.; Ševčík, S. Precursors of Hydrophilic Polymers, 5. Activation Parameters of Quaternization of Poly(2-Dimethylaminoethyl Methacrylate) with Methyl Iodide. Die Makromol. Chemie 1985, 186, 1657-1663.

(43) Hu, G.-H.; Wang, W. A Kinetic Model for Steric Hindrance Effects on Quaternization of Poly(Vinylpyridines). J. Polym. Sci. Part A Polym. Chem. 1993, 31, 3453-3464.

(44) Brittain, W. J.; Minko, S. A Structural Definition of Polymer Brushes. J. Polym. Sci. Part A Polym. Chem. 2007, 45, 3505-3512.

(45) Matsunaga, N.; Hori, M.; Nagashima, A. Gaseous Diffusion Coefficients of Methyl Bromide and Methyl Iodide into Air, Nitrogen, and Oxygen. Heat Transf. Res. 2009, 38, 361-369.

(46) Efimenko, K.; Genzer, J. How to Prepare Tunable Planar Molecular Chemical Gradients. Adv. Mater. 2001, 13, 1560-1563.

(47) Genzer, J.; Efimenko, K.; Fischer, D. A. Formation Mechanisms and Properties of Semifluorinated Molecular Gradients on Silica Surfaces. Langmuir 2006, 22, 8532-8541.

(48) Douglas, J. F.; Efimenko, K.; Fischer, D. A.; Phelan, F. R.; Genzer, J. Propagating Waves of Self-Assembly in Organosilane Monolayers. Proc. Natl. Acad. Sci. 2007, 104, 10324 LP $-10329$. 
TOC figure

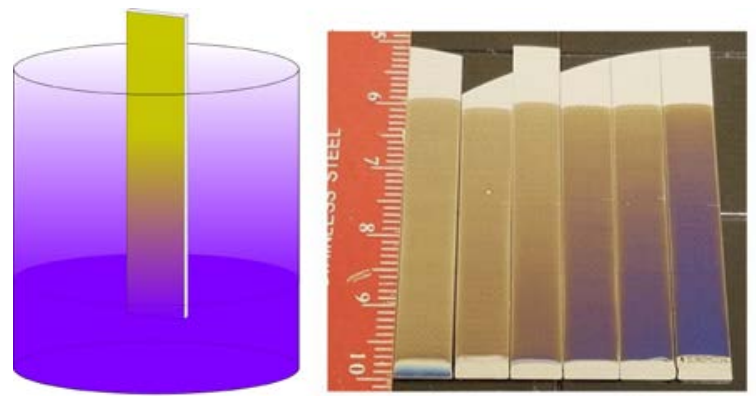




\title{
Supporting Information
}

Charge density gradients of polymer thin film by gaseous phase quaternization

\author{
Yeongun $\mathrm{Ko}^{1}$, Stephanie Christau ${ }^{2}$, Regine von Klitzing ${ }^{3}$, Jan Genzer ${ }^{1,4, *}$ \\ ${ }^{1}$ Department of Chemical and Biomolecular Engineering, North Carolina State University \\ Raleigh, NC 27695-7905, USA \\ ${ }^{2}$ Department of Chemical Engineering, Biointerfaces Institute, University of Michigan \\ Ann Arbor, MI 48109, USA \\ ${ }^{3}$ Department of Physics, Technische Universität Darmstadt \\ 64289 Darmstadt, Germany \\ ${ }^{4}$ Global Station for Soft Matter, Global Institution for Collaborative Research and Education \\ Hokkaido University, Sapporo, 060-0808, Japan
}

\section{Experimental Details}

\section{Materials}

All chemicals were purchased from Sigma-Aldrich and used as received unless noted otherwise. Deionized water (DIW) with resistivity >15 M $\mathbf{c m}$ was obtained from Millipore Elix 3. 2-(dimethylamino)ethyl methacrylate (DMAEMA) was passed through an inhibitor removal column (Aldrich) before polymerization. 11-(2-bromo-2-methyl)propionyloxy undecyltrichlorosilane (eBMPUS) was purchased from Gelest. Silicon wafers (p-doped, orientation <100>) were purchased from Silicon Valley Microelectronics.

\section{Preparing polymer thin films}

$15 \mathrm{~mL}$ (0.089 mol) of (2-dimethylaminoethyl) methacrylate (DMAEMA) and $5 \mathrm{~mL}$ of 1,4-dioxane were charged into a $25 \mathrm{~mL}$ vial. $146 \mathrm{mg}(0.89 \mathrm{mM})$ of AIBN was dissolved into the monomer solution, followed by argon gas purging for $30 \mathrm{~min}$. The vial was immersed in an oil bath at $70^{\circ} \mathrm{C}$ for 12 hours under constant argon gas purge. After the polymerization, the PDMAEMA was

*Corresponding author: jgenzer@ncsu.edu 
precipitated in hexanes and subsequently dissolved in ethanol, followed by passing through 0.2 $\mu \mathrm{m}$ filters. Silicon wafers $(6 \mathrm{~mm} \times 40 \mathrm{~mm}$ ) were exposed to ultraviolet/ozone treatment (UVO, Model 42, Jelight Co.) for 30 min before use. To deposit PDMAEMA films of variable thickness on the UVO-treated silicon substrates, we prepared solutions featuring 20,30, 45, and $60 \mathrm{mg} / \mathrm{mL}$ of PDMAEMA in ethanol, dipped the silicon wafers vertically into the respective solution and pulled the wafer out of the solution with a constant rate of $50-100 \mathrm{~mm} / \mathrm{min}$.

Films featuring grafted PDMAEMA chains were prepared by utilizing surface-initiated atom transfer radical polymerization (ATRP). The silane initiator (eBMPUS) was deposited on the silicon wafer (12 mm x $40 \mathrm{~mm}$ in size) by incubating in a solution $(0.005 \% \mathrm{v} / \mathrm{v}$ of eBMPUS in hexanes) at room temperature for 48 hours. The ATRP solution prepared with DMAEMA (4 mL, $23.7 \mathrm{mM}$ ), DIW (36.8 mL, $2.0442 \mathrm{M}$ ), and isopropanol (9.2 mL, $120.3 \mathrm{mM})$ in a $150 \mathrm{~mL}$ roundbottom flask followed by purging argon gas for 10 minutes. The ligand 2,2'-bipyridine (0.1421 g, $0.9 \mathrm{mM})$ and the catalyst $\mathrm{CuCl}(0.03917 \mathrm{~g}, 0.4 \mathrm{mM})$ were added to the flask while the solution was mixed using a stir-bar and degassed by argon gas for another 15 minutes. The PDMAEMA grafts were obtained by placing the initiator-coated substrates in a $20 \mathrm{~mL}$ vial filled with the ATRP solution for $120 \mathrm{~min}$ at room temperature.

Quaternization of PDMAEMA grafts $(6 \mathrm{~mm} \times 10 \mathrm{~mm})$ in the liquid phase was carried out by immersing PDMAEMA brushes into a solution comprising 0.8, 1.6, 3.2, and $6.4 \mathrm{M}$ methyl iodide (MI) in ethanol at room temperature for specific reaction times.

Quaternization of PDMAEMA non-grafted film in the gaseous phase was carried out by placing the PDMAEMA-coated silicon wafers vertically in an empty standard $50 \mathrm{~mL}$ glass beaker (inner diameter $4 \mathrm{~cm}$, height $5.5 \mathrm{~cm}$ ). $200 \mu \mathrm{L}$ of the prepared methyl iodide-ethanol solution was injected to the bottom of the beaker. After the desired time, the specimen was removed from the beaker and dried with a nitrogen gas stream.

\section{Characterization}

We utilized variable angle spectroscopic ellipsometry (VASE) (J.A. Woollam Co.) to measure the thickness and refractive index of polymer thin films. All samples were characterized at $100^{\circ} \mathrm{C}$ with two angles of incidence ( 60 and $65^{\circ}$ relative to the normal) and the wavelength ranging from 400 to $800 \mathrm{~nm} .{ }^{1}$ A hot stage (FP82HT, Mettler Toledo) connected to a central processor (FP90, 
Mettler Toledo) was placed on the VASE sample stage to carry out the high-temperature ellipsometry measurements. The acquired ellipsometry data were analyzed with WVASE32 software (J.A. Woollam Co.). Single Cauchy layer on top of $1.5 \mathrm{~nm} \mathrm{SiO}$ x layer and Si substrate were used to fit the experimental data.

Fourier-transform infrared spectroscopy (FTIR) spectra were collected by performing 128 scans with $4 \mathrm{~cm}^{-1}$ resolution in attenuated total reflection (ATR) mode with Ge crystal on a Nicolet 6700 spectrometer and analyzed with OMNIC software.

\section{Results}

We used Equation 2 to fit the experimental quaternization kinetics in the liquid phase (Figure 1a in the manuscript). Table S1 lists the fitting parameters and R-squared values.

Table S1. Fitting parameters and R-squared for data plotted in Figure 1a.

\begin{tabular}{c|cccc}
\hline \multirow{2}{*}{ Fitting parameters } & \multicolumn{4}{|c}{ Concentration of methyl iodide in solution (M) } \\
& 0.8 & 1.6 & 3.2 & 6.4 \\
\hline $\mathrm{A}(\mathrm{mol} \mathrm{\% )}$ & 84.373 & 84.373 & 84.373 & 84.373 \\
$k_{1 l}\left(\mathrm{~s}^{-1}\right)$ & 0.0123 & 0.0222 & 0.0386 & 0.0563 \\
$R^{2}$ & 0.98 & 0.99 & 0.99 & 0.99 \\
\hline
\end{tabular}

We prepared solutions of PDMAEMA (20 mL in volume) in ethanol with 20, 30, 45 and $60 \mathrm{mg} / \mathrm{mL}$ concentrations. We dip-coated each sample and removed it from the solution with a constant pulling speed (100 mm/min). Figure S1 displays the film thickness as a function of the PDMAEMA concentration in solution. Film thicknesses of all PDMAEMA films were measured at $100^{\circ} \mathrm{C}$ to reduce the effect of water sorption. The thickness at ambient conditions was determined using the method described in ref 1. 


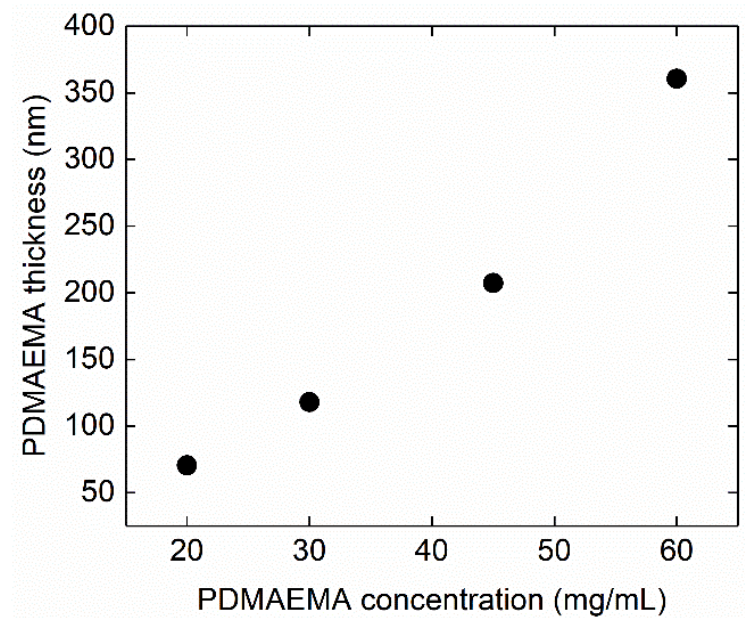

Figure S1. The dry thickness of PDMAEMA films at $100^{\circ} \mathrm{C}$ with different concentrations of PDMAEMA in ethanol solutions.

The tertiary amines in the DMAEMA repeat units are quaternized by reaction with MI resulting in poly(DMAEMA-co-qDMAEMA) random copolymers.

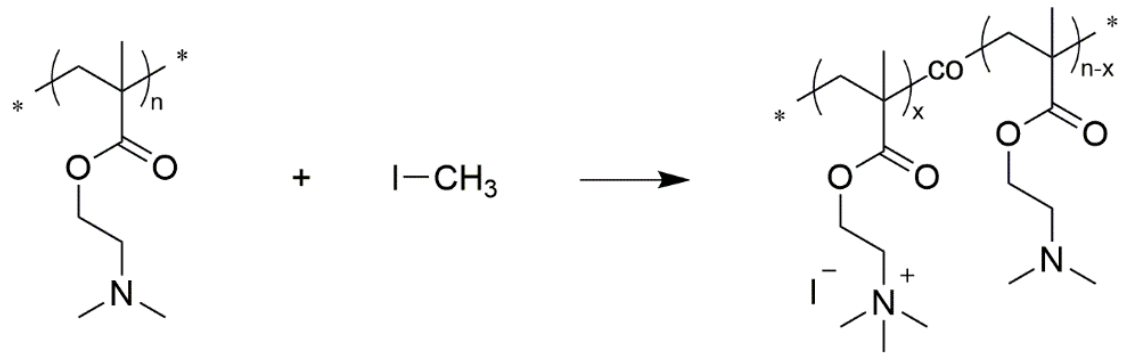

Scheme S1. Quaternization reaction scheme of PDMAEMA with methyl iodide (MI).

We normalized the band in the FTIR-ATR spectra for samples with variable DQ to the carbonyl vibration, whose intensity does not depend on DQ (Figure S2). The intensity bands that depict $\mathrm{N}^{+}$-C stretching vibrations (954 and $1481 \mathrm{~cm}^{-1}$ ) increase with increasing DQ while the vibration signal at $2775 \mathrm{~cm}^{-1}$, which corresponds to stretching of the $\mathrm{C}-\mathrm{H}$ bond attached to the tertiary amine, decreased with increasing DQ. The intensities of the signals mentioned above are plotted as a function of DQ in Figure S3. Besides, the intensity of the broad band at $\sim 3500 \mathrm{~cm}^{-1}$ increases with increasing DQ, indicating stronger water sorption at ambient conditions with increasing DQ. 


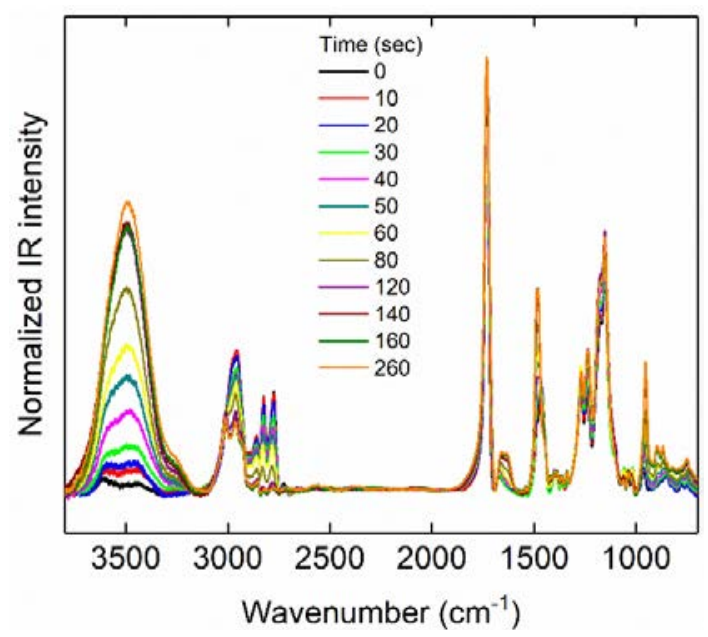

Figure S2. FTIR-ATR normalized absorption intensity of the carbonyl vibration $\left(1723 \mathrm{~cm}^{-1}\right)$ of quaternized PDMAEMA brushes as a function of increasing reaction time with MI in liquid phase reaction.

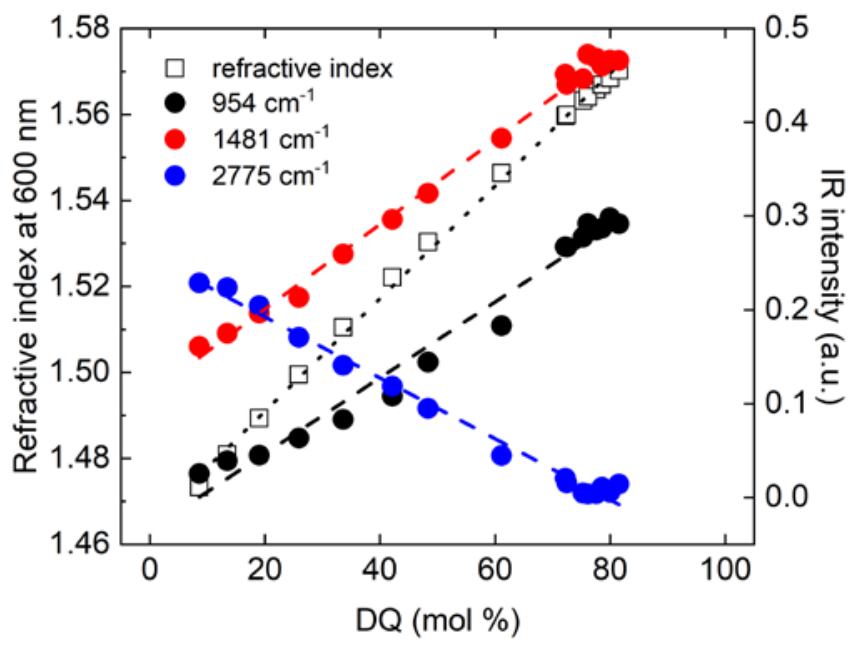

Figure S3. Refractive index at $600 \mathrm{~nm}$ (open square, left ordinate) and FTIR-ATR intensity (954 $\mathrm{cm}^{-1}$ : black circle, $1481 \mathrm{~cm}^{-1}$ : red circle, $2775 \mathrm{~cm}^{-1}$ : blue circle, right ordinate) normalized by the intensity at 1723 $\mathrm{cm}^{-1}$ of respective PDMAEMA brushes as a function of degree of quaternization (DQ, mol \%). The dashed lines are meant to guide the eye.

We have prepared a series of quaternized PDMAEMA in bulk and estimated DQ by using elemental analysis. Those films were spun cast on silicon wafers, and their refractive indices were determined by ellipsometry. We employed the effective medium approximation (EMA) to estimate DQ (vol \%) from the refractive indices of the copolymers. DQ (mol \%) was obtained from DQ (vol\%) and densities of PDMAEMA and quaternized PDMAMA (qPDMAEMA). The results plotted as the refractive index at $600 \mathrm{~nm}$ indicate that the optical properties of the films change 
linearly with DQ. We also analyzed the samples with Fourier-transform infrared spectroscopy (FTIR). All FTIR spectra were collected in attenuated total reflection (ATR) mode, followed by manual baseline correction and normalized by the intensity at $1723 \mathrm{~cm}^{-1}$, which does not depend on DQ. As shown in Figure S3, the IR intensities of the three distinct wavenumbers change linearly with DQ. The 954 and $1481 \mathrm{~cm}^{-1}$ signals show an increase with DQ, indicating $\mathrm{N}^{+}-\mathrm{C}$ stretching vibrations. ${ }^{2-4}$ The intensity at $2775 \mathrm{~cm}^{-1}$ corresponds to C-H stretching vibrations attached to tertiary amine decreases with increasing DQ. ${ }^{5}$ All IR intensities, and the refractive index change linearly with $\mathrm{DQ}$, as expected. ${ }^{6}$

In Figure S4 we plot the shift factors used to produce the master curves in Figure $\mathbf{4}$ and Figure 5. All values show a linear decrease with increasing the square root of process time. While we find a dependence of the shift factor on the concentration of MI in solution, no effect on the thickness of the PDMAEMA layer is found.
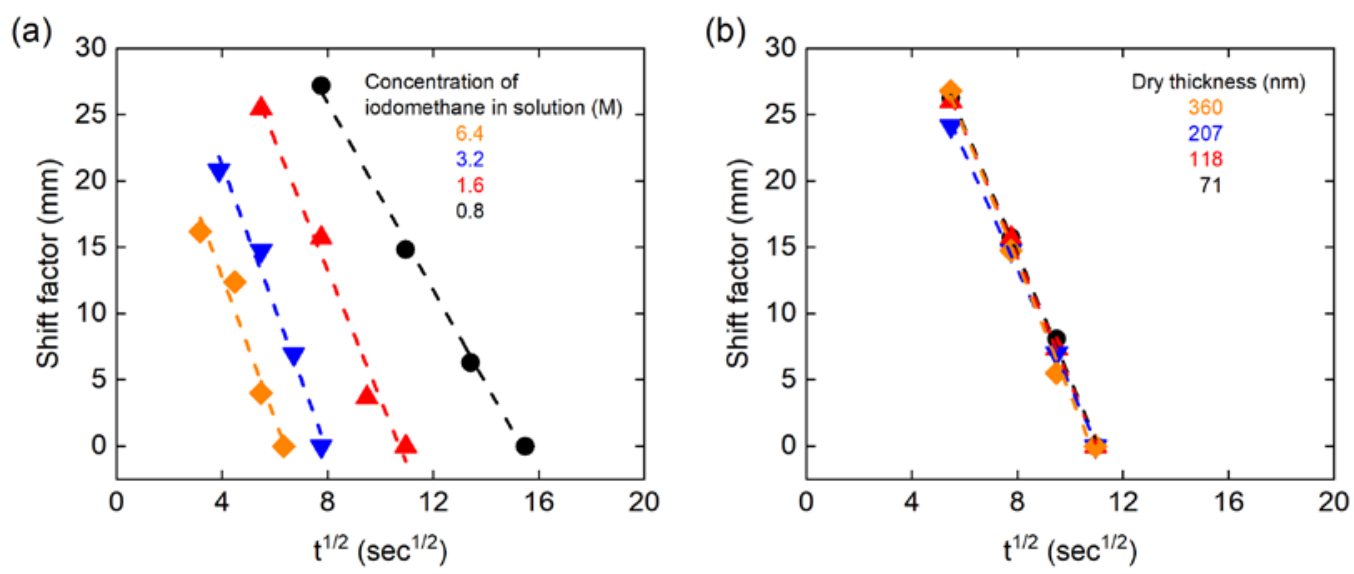

Figure S4. Shift factors used in Figure 3 and Figure 4 as a function of the square root of process time.

The diffusion source comprises MI molecules present in gas right above at the interface between the liquid and gaseous phases in a two-component methyl iodide/ethanol system, $c(0, t)$. To obtain an estimate for $c(0, t)$, we calculate the concentration of $\mathrm{MI}$ in the gaseous phase above the MI solution, $\left(c_{\text {gas,equil }}\right)$, by combining Raoult's law and the equation of state of a real gas. We first use Raoult's law to determine the mole fraction of MI in the gaseous phase $\left(y_{M I}\right)$ :

$$
p_{M I}^{*} \cdot x_{M I}=P \cdot y_{M I}
$$


where $P$ is the total pressure ( $=101.325 \mathrm{kPa}), p_{M I}^{*}$ is the vapor pressure of $\mathrm{MI}(=54.4 \mathrm{kPa})$, and $x_{I M}$ the mole fraction of MI in solution. Then we calculate the concentration of MI in the gaseous phase from the compressibility factor equation of state:

$$
c_{g a s, e q u i l}=y_{M I} \cdot \frac{P}{z \cdot R \cdot T}
$$

In Equation S2, $R$ is the universal gas constant, $T$ is absolute temperature, and $z$ is the compressibility factor. Using tabulated values for critical pressure (7366.33 kPa) and temperature (527.95K) for IM we calculate the reduced temperature and pressure at $T=298 \mathrm{~K}$ and $P$ listed above as $T_{r}=0.56$ and $P_{r}=0.014$, respectively. ${ }^{7}$ Using a compressibility chart, we estimate the compressibility factor to be $z \sim 0.96$. The results are displayed in Table S2.

Table S2. Calculations of methyl iodide concentration of gaseous phase in equilibrium state $\left(c_{\text {gas,equil }}\right)$. The $c_{\text {sol }}$ indicates the concentration of MI in solution.

\begin{tabular}{cccc}
\hline$c_{\text {sol }}(\mathrm{M})$ & $x_{M I}$ & $y_{M I}$ & $c_{\text {gas, equil }}(\mathrm{M})$ \\
\hline 0.8 & 0.047 & 0.025 & 0.0011 \\
1.6 & 0.094 & 0.051 & 0.0022 \\
3.2 & 0.190 & 0.102 & 0.0043 \\
6.4 & 0.385 & 0.207 & 0.0088 \\
16 & 1.000 & 0.537 & 0.0229 \\
\hline
\end{tabular}

There are a few caveats to this approach. First, we recognize that the system is not at equilibrium (where the Raoult's law applies) and that the mole fraction of methyl iodide in the vapor, as determined from Equation S1, constitutes only an upper estimate of the true value. We do not know the exact concentration of methyl iodide on the surface of the liquid. One may argue that there may be a small depletion of the solute because its surface tension $(25.8 \mathrm{mN} / \mathrm{m}$ as measured at $\left.43.5^{\circ} \mathrm{C}\right)$ is slightly higher than that of ethanol $\left(\sim 21.97 \mathrm{mN} / \mathrm{m}\right.$ as measured at $\left.25^{\circ} \mathrm{C}\right){ }^{8,9}$ However, evaporation may increase the MI local concentration at the liquid/vapor interface. The calculated values of $c_{\text {gas,equil }}$ are still useful because they help us estimate the upper limit of $c(0, t)$. Using the values of the rate constant of $k_{1 g}$ (cf. Figure $4 \mathbf{b}$ ) and the reported values of $c_{\text {gas,equil }}(\sim c(0, t)$ ), we estimate $k_{2 g} \sim 6.374 \pm 0.494(\mathrm{M}:)^{-1}$. This result indicates that the reaction kinetics in the gaseous 
phase is at least $\sim 650$ times faster than that in solution ( $c f$. Figure 1). In reality, the value of $k_{2 g}$ is likely higher than quoted because $c_{\text {gas,equil }}<c(0, t)$.

The height of the beaker reactor affects the steepness of the charge gradient ( $c f$. Figure S5). In addition to using the standard $50 \mathrm{~mL}$ beaker (inner diameter $4 \mathrm{~cm}$, height $5.5 \mathrm{~cm}$ ) we have carried out the same process using a $10 \mathrm{~mL}$ beaker (inner diameter $2.5 \mathrm{~cm}$, height $2.8 \mathrm{~cm}$ ) and obtained a narrower gradient that extended only over $\sim 2.5 \mathrm{~cm}$ across the substrate. Because the sample was taller than the beaker height, the substrate section positioned above the beaker was not quaternized (or quaternized to only a very small amount).
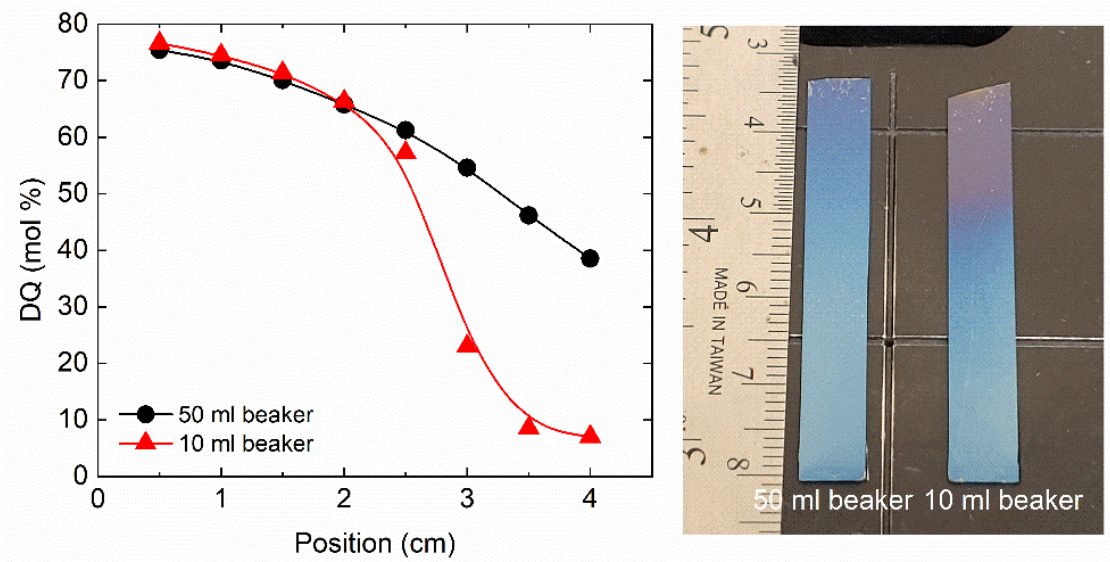

Figure S5. Charge gradients on PDMAEMA brushes generated from $50 \mathrm{~mL}$ beaker (black circle) and 10 $\mathrm{mL}$ beaker (red triangle), respectively, and the corresponding sample images on the right side. $200 \mu \mathrm{l}$ of 6.4 M methyl iodide in ethanol solution was used. The process time was $60 \mathrm{sec}$. The lines are meant to guide the eye.

\begin{tabular}{|c|c|c|c|c|}
\hline \multicolumn{4}{|c|}{$\begin{array}{l}\mathrm{C}_{\mathrm{MI}} \text { in solution } \\
\text { (M) }\end{array}$} & \multirow[t]{2}{*}{$\begin{array}{l}\text { Time } \\
\text { (sec) }\end{array}$} \\
\hline 6.4 & 3.2 & 1.6 & 0.8 & \\
\hline \multirow[t]{2}{*}{$\nabla$} & & & & 10 \\
\hline & $\Delta$ & & & 15 \\
\hline$\nabla$ & & & & 20 \\
\hline$\forall$ & 4 & $\oplus$ & & 30 \\
\hline \multirow[t]{7}{*}{$\not \supset$} & & & & 40 \\
\hline & $\Delta$ & & & 45 \\
\hline & 4 & (1) & $\square$ & 60 \\
\hline & & $\theta$ & & 90 \\
\hline & & - & a & 120 \\
\hline & & & I & 180 \\
\hline & & & D & 240 \\
\hline
\end{tabular}

Figure S6. Legend to symbol types in Figure 4 in the main text. $\mathrm{C}_{\mathrm{MI}}$ is the concentration of methyl iodide. 


\begin{tabular}{|c|c|c|c|c|}
\hline \multicolumn{4}{|c|}{$\begin{array}{l}\text { Dry thickness } \\
(\mathrm{nm})\end{array}$} & \multirow[t]{2}{*}{$\begin{array}{l}\text { Time } \\
\text { (sec) }\end{array}$} \\
\hline 360 & 207 & 118 & 71 & \\
\hline$\nabla$ & $\Delta$ & $\bullet$ & घ & 30 \\
\hline$\nabla$ & $\Delta$ & 0 & 口 & 60 \\
\hline$\forall$ & 4 & $\oplus$ & 田 & 90 \\
\hline$\not X$ & $\not$ & $\otimes$ & $\otimes$ & 120 \\
\hline
\end{tabular}

Figure S7. Legend to symbol types in Figure 5 in the main text.

\section{References}

(1) Ko, Y.; Miles, J. R.; Genzer, J. Determining Water Sorption and Desorption in Thin Hydrophilic Polymer Films by Thermal Treatment. ACS Appl. Polym. Mater. 2019, 1, 2495-2502.

(2) Donegan, M.; Dowling, D. P. Protein Adhesion on Atmospheric Plasma Deposited Quaternary Ammonium Salt Coatings. Plasma Process. Polym. 2013, 10, 526-534.

(3) Badawy, M. E. I.; Rabea, E. I.; Taktak, N. E. M. Antimicrobial and Inhibitory Enzyme Activity of N-(Benzyl) and Quaternary N-(Benzyl) Chitosan Derivatives on Plant Pathogens. Carbohydr. Polym. 2014, 111, 670-682.

(4) Hu, X.; Lin, X.; Zhao, H.; Chen, Z.; Yang, J.; Li, F.; Liu, C.; Tian, F. Surface Functionalization of Polyethersulfone Membrane with Quaternary Ammonium Salts for Contact-Active Antibacterial and Anti-Biofouling Properties. Materials 2016, 9, 376-387.

(5) Özçam, A. E.; Roskov, K. E.; Spontak, R. J.; Genzer, J. Generation of Functional PET Microfibers through Surface-Initiated Polymerization. J. Mater. Chem. 2012, 22, 58555864.

(6) Jhon, Y. K.; Semler, J. J.; Genzer, J. Effect of Solvent Quality and Chain Confinement on the Kinetics of Polystyrene Bromination. Macromolecules 2008, 41, 6719-6727.

(7) Weast, R. C. CRC Handbook of Chemistry and Physics, 67th ed.; CRC press: Fl, 1986; pp F-63.

(8) Weast, R. C. CRC Handbook of Chemistry and Physics, 67th ed.; CRC press: Fl, 1986; pp F-34.

(9) Haynes, W. M. CRC Handbook of Chemistry and Physics, 95th ed.; CRC Press: FL, 2014; pp 6-182. 